\title{
MECHANISMS LINKING ORAL HEALTH AND FRAILTY IN OLDER ADULTS: A NARRATIVE REVIEW
}

\author{
Kalliopi Konstantopoulou ${ }^{1 a^{*}(\mathbb{D})}$, Anastassia Kossioni ${ }^{16}$ (1) \\ 'Department of Prosthodontics, Dental School, National and Kapodistrian University of Athens, Athens, Greece \\ DaDDS, MSc; e-mail: kakonsta@dent.uoa.gr; ORCIDiD: https://orcid.org/0000-0002-4314-9222 \\ DDS, MSc, PhD, Professor; e-mail: akossion@dent.uoa.gr; ORCIDiD: https://orcid.org/0000-0003-0610-6984
}

Background Frailty is a geriatric syndrome in which multiple systems lose their physiological reserves resulting in increased vulnerability to stressors and risk of adverse health-related outcomes. There is an increasing number of studies discussing the association of oral health with frailty through several pathways. Objective The aim of this review was to describe the possible mechanisms linking oral health and frailty. Data Sources A narrative review was performed with literature search in PubMed, Google Scholar and ScienceDirect electronic databases. Reference lists from relevant studies and cited papers were also investigated.

Study Selection The review included full papers of any study design, published in peer-reviewed journals in English until July 2021.

Data Extraction Current literature indicates four possible mechanisms linking oral health and frailty.

Data Synthesis The first mechanism refers to the nutritional pathway. It is reported that poor oral health negatively affects protein and vitamins intake. Malnutrition and decreased energy intake have a dominant role in frailty onset. Inflammation is another mechanism. Periodontal disease causes a systemic increase of pro-inflammatory biomarkers which in turn may lead to muscle strength deterioration. Furthermore, oral health can be related to frailty through neural mechanisms. Specifically, occlusion and proprioception from the periodontal ligament contribute to the control of body balance. Finally, the mechanisms include the psychological pathway, since poor oral health may lead to social isolation and depression which increase the risk of negative general health outcomes. More studies are necessary to clarify the previous associations and reveal any causative effects.

\section{KEYWORDS}

Oral Health; Frailty; Malnutrition; Inflammation; Depression.

\section{INTRODUCTION}

One of the dominant characteristics of the older population is heterogeneity, therefore overall health and function can vary substantially among individuals of the same chronological age. The concept of frailty has come to the forefront of research interest in Geriatric Medicine as a geriatric syndrome characterized by multiple functional impairments trying to explain this diversity among older people $[1,2,3]$. Frailty is defined as a clinical state of increased vulnerability related to ageing in which multiple systems lose their physiological reserves and the homeostatic balance is disrupted, resulting in an increased risk of adverse health-related outcomes, including functional impairment, dependency, hospital admission, institutionalization, reduced quality of life and mortality, even after exposure to a minor stressor, such as a viral infection or the use of a new drug $[2,4]$. The most common clinical manifestations of frailty are falls, confusion, functional decline and several non-specific signs and symptoms, such as unintentional weight loss, extreme fatigue and frequent infections. Genetic and environmental factors combined with epigenetic mechanisms are closely associated to the cumulative molecular and cellular damage, and pathophysiology of frailty.

As frailty is considered a dynamic and modifiable condition exercise (aerobic, balance and resistancebased), caloric and protein support, vitamin D intake and reducing polypharmacy seem to be effective strategies in its prevention or even reversion [4].

Prevalence of frailty among community-dwelling older adults and nursing home residents is estimated to be approximately $16.7 \%$ [5] and $52.3 \%$ respectively [6]. Regarding the assessment of frailty, a total of 67 instruments are available in the literature [7]. The Physical Frailty Phenotype has been identified as the most widely used instrument [7]. 
According to this instrument, frailty is diagnosed when at least three of the following components are met: unintentional weight loss, self-reported exhaustion, low physical activity, weak hand grip strength and slow gait speed, while pre-frailty is defined by the presence of one or two of these criteria [8]. Moreover, the Clinical Frailty Scale and the Frailty Index of Accumulative Deficits are among the nine most-referenced frailty instruments [7]. It was developed as a 7-point ordinal scale and has been modified as a 9-point scale from one (very fit) to nine (terminally ill with a life expectancy of less than six months) based on information about health status derived from medical history and clinical examination; a score of 5 or more indicates frailty [9]. The Frailty Index of Accumulative Deficits is based on the multi-dimensional nature of frailty and is expressed as a ratio of various accumulated health deficits [10].

Frailty has come to the forefront of the research interest in the dental field as well. Poor oral health has a high prevalence among older adults and there is an increasing number of studies which demonstrate cross-sectional, as well as longitudinal associations of oral health indicators with frailty or its components through several suggested pathways [11-13]. A systematic review showed a relationship between aspects of oral health, such as number of teeth, need for and use of dental prostheses, and frailty or pre-frailty, and suggested the existence of various mediators of this association which should be further investigated [11]. In a systematic review of longitudinal studies, the number of teeth, oral function, accumulation of oral health problems and number of dry mouth symptoms were identified as predictors of frailty [12]. The evident associations between oral health and frailty status among older people suggest that the integration of frailty assessment into dental treatment planning might be useful in providing the most appropriate dental care and preventive strategies to older adults who are at a state of increasing vulnerability [13]. Moreover, oral health, and particularly occlusal force and mastication, have been associated with sarcopenia [14], a muscle disease characterized by decreased muscle strength and muscle mass, and/ or reduced physical performance, which has many mutual clinical features with frailty and may serve as a precursor of frailty $[15,16]$.

Apart from the various individual oral health indicators that have been investigated in relation to frailty, the term "oral frailty" has been introduced by the Japanese Society describing a condition of decreased articulation, slight choking, or spillage while eating and increased number of unchewable foods [17]. A longitudinal study in Japanese community-dwelling older adults, describing oral frailty as the presence of at least three of the following parameters: less than 21 natural teeth, decreased masticatory performance, decreased oral diadochokinesis for the syllable "ta", decreased tongue pressure, subjective difficulties in eating tough foods and swallowing tea or soup showed that it may predict new onsets of frailty as defined by the Physical Frailty Phenotype and mortality [18].

As an increasing number of studies has shown an association between oral health and frailty, the purpose of the present narrative review was to describe the possible linking mechanisms.

\section{METHODOLOGY}

A literature search in PubMed, Google Scholar and ScienceDirect electronic databases was performed. The following keywords were used: (oral health OR oral function) AND (frailty) AND (linking mechanisms OR linking pathways). In addition, reference lists from relevant studies and cited papers were investigated. The titles and abstracts of the retrieved articles were screened to decide whether full-text reading was required, and full texts were retrieved for the selected articles. Included studies should have been published in peer-reviewed journals in English language, while no limits were set on the study design and the year of publication. Articles published until July 2021 were included in the present review.

\section{RESULTS}

Current literature indicates four possible mechanisms through which oral health is associated to frailty: a) nutritional pathway, b) inflammation, c) neural mechanisms and d) psychological pathway (Fig. 1).

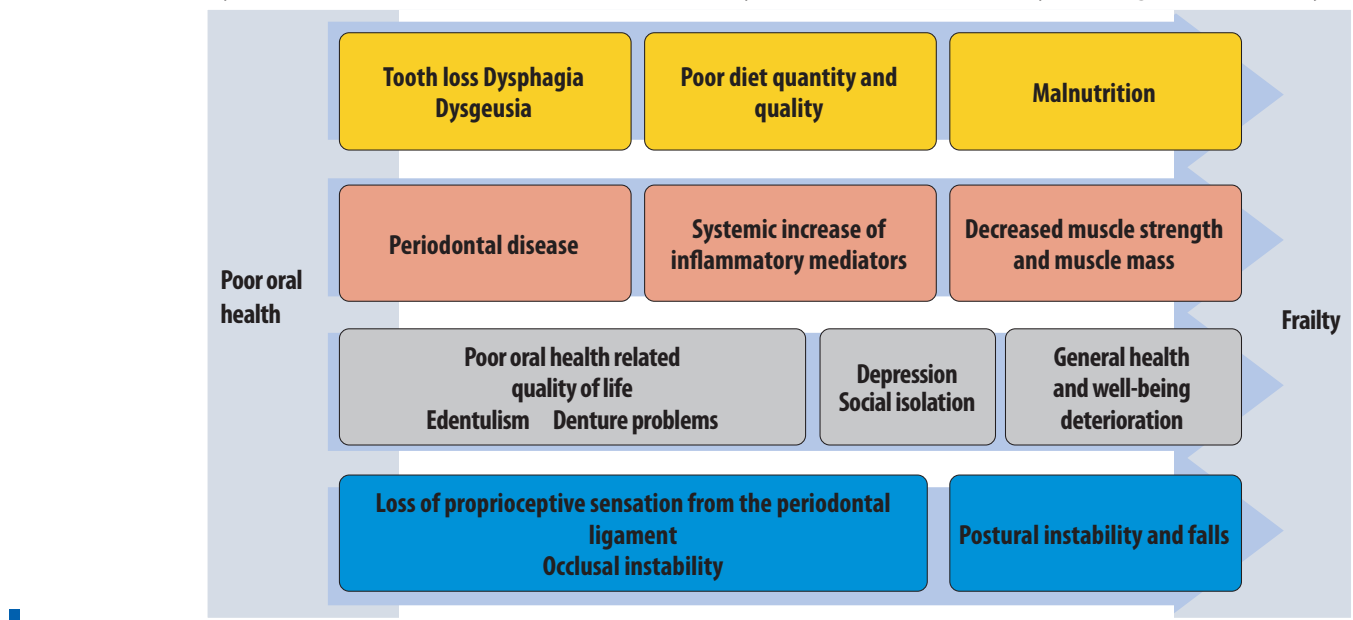

Figure 1. Screening process. 


\subsection{Nutritional pathway}

The first linking mechanism refers to the nutritional pathway and there is an interesting discussion on the association between oral health and nutrition.

Malnutrition is associated with frailty $[19,20]$. A metaanalysis revealed that a total of $68 \%$ of communitydwelling older adults with malnutrition had frailty [21].

Another meta-analysis demonstrated the substantial association between malnutrition and frailty or sarcopenia, with the co-occurrence of two or all the afore-mentioned conditions in about $50 \%$ of the hospitalized older adults [22].

There are indications that energy intake, as well as nutrient quality have an important role in the onset of frailty and the pathogenesis of sarcopenia [23]. Deficiencies in nutrients have been associated with mitochondrial dysfunction which in turn may cause fatigue and weakness, two elements of Physical Frailty Phenotype [24,25]. Furthermore, a decreased amount of protein intake possibly contributes to the pathogenesis of frailty findings, based on a meta-analysis of observational studies suggesting an association between an increased intake of dietary protein and a lower prevalence of frailty $(O R=0.67)$ [26]. In addition to the quantity of proteins, other parameters, such as protein source and protein distribution across meals, may also have an impact on the development and progression of frailty in older people [18].

Diet quality overall has been associated with a lower incidence of frailty in older adults $[27,28]$. Regarding the eating patterns in older adults, higher adherence to the Mediterranean Diet, which is characterized by a high intake of plant foods and olive oil and low consumption of red meats, have been inversely correlated to the loss of muscle mass, sarcopenia and frailty [29]; a meta-analysis demonstrated the protective role of the Mediterranean Diet against physical disability $(\mathrm{OR}=0.75)$ and frailty $(\mathrm{OR}=0.42)$ [30]. It is hypothesized that the Mediterranean Diet has a protective action on skeletal muscle health (myoprotective effect), since it is regarded as a source of bioactive nutrients and has anti-oxidative and anti-inflammatory properties [31]. On the other hand, Westernised dietary patterns, characterized by high consumption of refined cereals, whole dairy products, and processed meat, have a direct relationship with the increased risk of developing several components of physical frailty phenotype, namely weight loss and slow gait speed [32].

Poor dentition, dysphagia and dysgeusia are considered to be among the nine common risk factors (the nine d's) of malnutrition in older adults [33], but more research is necessary to clarify any causative effects and the role of individual contributors.

Edentulism and the presence of less than 21 natural teeth have been associated with the decreased intake of fruits, vegetables and proteins and the increased consumption of carbohydrates [34]. A meta-analysis showed that a lower number of remaining teeth was associated with poorer nutritional status, while edentulism and the presence of a prosthesis were not found to have a statistically significant effect on malnutrition in older adults [35].

However, a systematic review of longitudinal studies has shown that the evidence of a causative effect between tooth loss and nutritional status and nutrient intake was minor [36]. Also, the results of a randomized controlled trial indicated that both removable partial dental prostheses and the shortened dental arch concept generated equal improvement in partially dentate older adults' masticatory performance, but the masticatory performance could not predict nutritional status, due to their weak association [37]. Impaired masticatory performance in older adults is affected by a variety of factors and may lead to alteration of dietary choices and, subsequently, to malnutrition [38]. Better masticatory performance among functionally independent older adults, was independently associated with higher adherence to the Mediterranean Diet, in contrast to other dental indicators such as the number of natural teeth and use of removable prostheses [39], while older Greeks were more adherent to the components of the Mediterranean Diet compared to younger ones disregarding their dental status [40]. The enhancement of the maximum bite force and masticatory performance has been viewed as a prerequisite for a normal nutritional status in older people $[41,42]$. Nevertheless, the prosthetic rehabilitation of missing teeth does not seem sufficient for the treatment of malnutrition, and should be accompanied by nutritional advice, as this combination has been shown to lead to alterations in the food intake such as fruits and vegetables and to improvement of older adults' nutritional status [41-44].

Regarding dysgeusia, taste alteration leads to a diminution of food enjoyment, which in turn may decrease appetite and result in malnutrition [45]. Dysphagia (swallowing problems), which is highly prevalent in older individuals, can alter the oral intake of foods and liquids and, thus, serves as a risk factor of malnutrition [46-48]. According to a systematic review, chewing and swallowing problems were among the factors which were consistently associated with poor nutrition in nursing home residents $[31,49]$. Furthermore, high Xerostomia Index scores in older adults have been associated with the selective avoidance of foods including raw carrots, whole apples and nuts, lettuce, corn and grilled or fried meats [50].

In a cross-sectional study, community dwelling older adults with oral frailty (based on the definition proposed by Tanaka et al.) were found to have a greater possibility of more severe malnutrition assessed using the Mini Nutritional Assessment - Short Form $(\mathrm{OR}=2.17)$ and the level of serum albumin $(\mathrm{OR}=1.59)$ [51].

Also, a two-year longitudinal study demonstrated that oral frailty was associated with an increased risk of deterioration of nutritional status $(O R=2.24)$ [52]. 
There are indications that oral health status is independently associated with malnutrition [53]. However, the findings of a systematic review of prospective studies regarding dental status and swallowing function as determinants of malnutrition in older adults were inconsistent, while moderate quality evidence proposed that periodontal diseases, oral pain and difficulties in mastication are not determinants of malnutrition [54]. Therefore, mastication seems to explain only part of variance in the intake of food and/or nutrients in independent older adults [41], and more high-quality studies are necessary.

\subsection{Inflammation}

The term "inflammaging" describes an agerelated state of low-grade chronic inflammation. It is characterized by both increased levels of proinflammatory cytokines, such as CRP and IL- 6 and acute phase proteins, such as CRP, and decreased concentrations of IL-10, which lead to deterioration of immunological homeostasis [55, 56]. Thus, inflammaging has been assumed as an underlying mechanism of frailty. Proinflammatory biomarkers, principally interleukin 6 (IL-6) and tumor necrosis factor-a (TNF-a) can have an effect on the onset of frailty [57]. The results of a meta-analysis demonstrated a cross-sectional association between higher concentrations of CRP and IL-6, and frailty and pre-frailty, while no statistically significant longitudinal relationship emerged [58]. Another meta-analysis revealed a cross-sectional quantitative relationship between immunological biomarkers and frailty among older adults assessed with Physical Frailty Phenotype, which was stronger for CRP and IL-6 and weaker for TNF-a [56].

In a prospective cohort study, elevated serum levels of IL-6 acted as a predictor of walking speed decline among community-dwelling older adults [59]. Also, the findings of a meta-analysis suggested that higher levels of circulating CRP, IL- 6 and TNF-a were significantly associated with lower handgrip strength and knee extension strength, and CRP levels were significantly inversely correlated to skeletal muscle mass [60]. A meta-analysis of cross-sectional studies showed that serum levels of CRP were elevated in people with sarcopenia compared to controls, while no statistically significant associations were found between serum IL- 6 levels and TNF-a levels, and sarcopenia [61].

There is evolving evidence of strong associations between periodontitis, low-grade inflammation and systemic health. The accumulation of periodontopathogenic bacteria into the gingival sulcus stimulates a local inflammatory response and pro-inflammatory mediators such as interleukin 6 (IL6) and tumor necrosis factor a (TNF-a) are produced in periodontal lesions, which may move into the systemic circulation. Also, serum levels of C-reactive protein (CRP), an acute phase reactant produced mainly in the liver in various inflammatory cytokines, are elevated in patients with periodontitis [62].

\subsection{Psychological pathway}

Late-life depression and frailty have been described as comorbid geriatric syndromes [63]. A metaanalysis supported a reciprocal relationship between depression and frailty in older adults [64]. A systematic review suggested the strong prospective relationship between the presence of depression and greater risk of incident frailty among community-dwelling older adults [65]. Also, a strong correlation between depression and risk of frailty was found in a meta-analysis and this risk was higher for older men compared to older women (OR=4.76 and $O R=2.25$ respectively) [66]. The findings of another meta-analysis revealed a weak negative correlation between depressive symptomatology and handgrip strength [67]. Furthermore, the results of a longitudinal study demonstrated that both loneliness and social isolation were independently associated with incident frailty [68]. Another prospective cohort study showed a negative association between frequency of laughter and risk of functional disability among older individuals [69].

On the other hand, a longitudinal study showed that high levels of loneliness were associated with increased risk of incident physical frailty, but no relationship was found between loneliness and social isolation, and rate of change in frailty index [70]. Also, the results of a population-based cohort study suggested that depression does not act as a risk factor for the onset of frailty in older people [71]. The bidirectional association between depression and frailty is questioned, but it is indicated that they share some common risk factors in the short and long term [72].

Oral health has an effect on various aspects of older adults' quality of life among which are appearance and socializing [73]. Poor oral health may have negative consequences on social interaction and self-esteem and may lead to depression, which has an adverse effect on general health and wellbeing $[74,75]$. Cross-sectional as well as longitudinal associations between oral health related quality of life and loneliness in older adults have been identified [76].

Tooth loss can negatively affect speech and appearance, while denture problems may result in embarrassment due to dislodgement, pain and discomfort [38,77]. Older adults with fewer natural teeth and those who do not use dentures may have a greater possibility of social isolation [78]. In addition, tooth loss and selfreported dry mouth were found to be associated with a higher risk of developing depression among older adults in a longitudinal study [79]. Tooth loss and selfreported oral health problems, such as difficulty in chewing tough foods, may be longitudinally correlated to development or worsening of depression in older adults [80]. 
Also, a significant association was found between lower GOHAI (Geriatric Oral Health Assessment Index) scores and depression in Greek community-dwelling older adults [81]. A meta-analysis revealed a positive association of edentulism (OR=1.28) and periodontal disease $(H R=1.73)$ with depression in adults and older people [82].

A population-based cross-sectional study indicated a bidirectional association between the number of remaining teeth and the frequency of laughter among community-dwelling older adults [83].

Moreover, the results of a nationwide populationbased cohort study demonstrated that periodontitis was associated with the increased risk of subsequent depression [84].

On the other hand, a study in older Greeks has shown that the frequency of eating out was not affected by the dental status, but other biological and social factors played a more significant role indicating the multifactorial nature of human behaviour [85].

\subsection{Neural mechanisms}

The deterioration of body balance control is one of the most common causes of falls among older people [86]. Evidence has shown the existence of a positive association between postural instability and frailty or pre-frailty $[87,88]$. In a meta-analysis, an association between falls and frailty $(\mathrm{OR}=1.80)$ was found [89]. Moreover, a systematic review and metaanalysis provided a significant association between frailty and the future risk of falls among communitydwelling older adults, greater in males [90]. Another systematic review and meta-analysis with similar findings showed that community-dwelling older adults with frailty and prefrailty were at higher risk for falls and, also, those with frailty were more prone to recurrent falls [91].

Oral health may be related to postural stability through neural mechanisms. The stomatognathic system plays a role in the control of body posture [92]. Dental occlusion, trigeminal afferents and proprioception from the periodontal ligament may contribute to the control of postural stability, therefore tooth loss is a possible risk factor for postural instability $[92,93]$.

A case-control study showed that edentulous older adults had statistically significantly less body balance activity and higher body oscillation compared to those who maintained their dentition, while the use of complete denture(s) was not associated with body balance control [93]. However, in another study, complete dentures were found to have a positive effect on static and dynamic stability among edentulous older individuals [94]. In a longitudinal study, the complete loss of occlusion with natural teeth (Eichner index $\mathrm{C}$ ) was associated with decreased one-leg standing time with eyes open ( $\mathrm{OR}=4.27)$ [95]. Furthermore, a prospective study disclosed an independent association of maximum occlusal force with one-leg standing time among men aged 85 and older [96]. The results of a cohort study showed a strong relationship between occlusal status and postural stability [97]. According to a prospective cohort study, older adults with fewer than 20 teeth and those not using dentures were at higher risk for incident falls (OR=2.5) [98].

Also, the presence of teeth $(\mathrm{OR}=0.59)$ and dentures $(\mathrm{OR}=0.66)$ was significantly associated with decreased risk of falls in older adults with dementia [99]. On the other hand, the findings of a crosssectional study demonstrated that the use of dentures was correlated to decreased balance in both static and dynamic conditions and the duration of denture use was negatively asso-ciated with dynamic balance [100].

\section{DISCUSSION}

Current evidence supports both the cross-sectional and the longitudinal association between oral health and frailty [11-13], but more studies are necessary to confirm the evidence and clarify any causative effects. The majority of studies investigating this relationship were conducted in community dwelling older adults in Japan and the most commonly used frailty assessment instrument was the Physical Frailty Phenotype. This review has also identified four potential pathways which may link oral health and frailty that also need further investigation: the nutritional pathway, inflammation, the psychological pathway and neural mechanisms.

Oral health indicators, such as the number of teeth, masticatory performance, swallowing problems and dysgeusia seem to contribute to the nutritional status in older adults [33-35,38,45-52]. However, robust evidence on the association between oral health and malnutrition is still lacking as various confounding factors are implicated $[41,42,54]$. Malnutrition and decreased energy intake, in turn, are considered to have a dominant role in the onset of frailty and the pathogenesis of sarcopenia [23-26]. The multifactorial nature of nutritional status and food choices has been acknowledged, therefore, the interdisciplinary collaboration between dental professionals, dietetics practitioners, and primary care providers is necessary for treatment and prevention of malnutrition [42].

Regarding the pathway of inflammation, periodontitis may lead to systemic increase of inflammatory mediators, which may serve as an underlying mechanism of frailty. Nevertheless, the findings regarding the association of periodontitis with frailty are contradictory and evidence which supports inflammation as a linking mechanism between oral health and frailty is weak [12].

There are indications that poor oral health, namely tooth loss, periodontitis and self-assessed oral health status negatively influences older adults' quality of life and is associated with an increased risk of depression in older adults $[78-82,84]$. 
Reciprocal, as well as prospective associations between depre-ssive symptomatology and frailty in older adults havebeen reported $[64,65]$, while it is suggested that depression and frailty are affected by common causes such as biological, psychosocial, behavioral, and environmental factors [72].

As far as the neural mechanisms are concerned, the literature supports the existence of a relationship between the stomatognathic system and body posture; the loss of proprioception from periodontal ligament may have a negative impact on the head position and contribute to postural instability [92,93]. posture; the loss of proprioception from periodontal ligament may have a negative impact on the head position and contribute to postural instability [92,93]. Deterioration in control of body balance is one of the leading causes of falls in older people [86]. Falls, in turn, are associated with frailty [87-89] and are among the most common clinical manifestations of frailty [90,91]. This narrative review has revealed the need for further research including case-control and randomized controlled trials to thoroughly explore the association between oral health and frailty and also the specific role of oral health as a predictor

\section{REFERENCES}

1. Ahmed N, Mandel R, Fain MJ. Frailty: an emerging geriatric syndrome. Am J Med. 2007;120(9):748-753. doi: 10.1016/j. amjmed.2006.10.018. PMID: 17765039.

Full text links PubMed Google Scholar

2. Clegg A, Young J, Iliffe S, Rikkert MO, Rockwood K. Frailty in elderly people. Lancet. 2013;381(9868):752-762. doi: 10.1016/ S0140-6736(12)62167-9 PMID: 23395245; PMCID: PMC4098658. Free PMC article PubMed Google Scholar WoS

3. Mitnitski AB, Graham JE, Mogilner AJ, Rockwood K. Frailty, fitness and late-life mortality in relation to chronological and biological age. BMC Geriatr. 2002;2:1. doi: 10.1186/1471-2318-2-1. PMID: 11897015; PMCID: PMC88955.

Full text links Free PMC article PubMed Google Scholar WoS 4. Morley JE, Vellas B, van Kan GA, et al. Frailty consensus: a call to action. J Am Med Dir Assoc. 2013;14(6):392-397. doi: 10.1016/j. jamda.2013.03.022. PMID: 23764209; PMCID: PMC4084863 Full text links Free PMC article PubMed Google Scholar WoS 5. Ofori-Asenso R, Chin KL, Mazidi M, et al. Global incidence of frailty and prefrailty among community-dwelling older adults: a systematic review and meta-analysis. JAMA Netw Open. 2019;2(8):e198398. doi: 10.1001/jamanetworkopen.2019.8398. PMID: 31373653; PMCID: PMC6681553.

Full Text links Free PMC article PubMed Google Scholar WoS 6. Kojima G. Prevalence of Frailty in Nursing Homes: A systematic review and meta-analysis. J Am Med Dir Assoc. 2015;16(11):940945. doi: 10.1016/j.jamda.2015.06.025. PMID: 26255709. Full text links PubMed Google Scholar WoS

7. Buta BJ, Walston JD, Godino JG, et al. Frailty assessment instruments: systematic characterization of the uses and contexts of highly-cited instruments. Ageing Res Rev. 2016;26:53-61. doi: 10.1016/j.arr.2015.12.003. PMID: 26674984; PMCID: PMC4806795. Full text links Free PMC article PubMed Google Scholar WoS 8. Fried LP, Tangen CM, Walston J, et al. Frailty in older adults: evidence for a phenotype. J Gerontol A Biol Sci Med Sci. 2001;56(3):M146-M156. doi: 10.1093/gerona/56.3.m146. PMID: 11253156.

Full text links PubMed Google Scholar WoS

9. Moorhouse P, Rockwood K. Frailty and its quantitative clinical evaluation. J R Coll Physicians Edinb. 2012;42(4):333-340. doi: 10.4997/JRCPE.2012.412. PMID: 23240122.

Full text links PubMed Google Scholar WoS

10. Mitnitski AB, Mogilner AJ, Rockwood K. Accumulation of deficits as a proxy measure of aging. Sci. World J. 2001;1:323-336. doi: 10.1100/tsw.2001.58. PMID: 12806071; PMCID: PMC6084020. Full text links $\underline{\text { Free PMC article PubMed Google Scholar WoS }}$ and marker of frailty. Moreover, more studies are necessary to explore the potential linking mechanisms including nutrition, inflammation, psychological and neural pathways. Studies in community-dwelling older individuals and nursing home residents should also examine the possible protective effect of systematic oral care provision against frailty. Statistically significant results on these parameters may lead to the integration of markers of oral health in the frailty assessment instruments and to the development of appropriate preventive strategies in the context of oral health promotion.

\section{CONCLUSIONS}

Oral health and frailty may be linked through nutritional, inflammatory, psychological and neural mechanisms. Further studies are necessary to thoroughly elucidate the role of these pathways as mediators of the latter association and, subsequently, to determine the most effective preventive strategies.

\section{AUTHOR CONTRIBUTIONS}

KK: protocol, data gathering, data analysis, authoring the draft. AK: concept, protocol, critically revising the manuscript.

11. Tôrres LH, Tellez M, Hilgert JB, et al. Frailty, Frailty components, and oral health: a systematic review. J Am Geriatr Soc. 2015;63(12):2555-2562. doi: 10.1111/jgs.13826. PMID: 26563844.

Full text links PubMed Google Scholar WoS

12. Hakeem FF, Bernabé E, Sabbah W. Association between oral health and frailty: a systematic review of longitudinal studies. Gerodontology. 2019;36(3):205-215. doi: 10.1111/ger.12406. PMID: 31025772.

Full text links PubMed Google Scholar WoS

13. Slashcheva LD, Karjalahti E, Hassett LC, et al. A systematic review and gap analysis of frailty and oral health characteristics in older adults: a call for clinical translation. Gerodontology. 2021;10.1111/ger.12577. doi: 10.1111/ger.12577. PMID: 34331353.

Full text links PubMed Google Scholar

14. Hatta K, Ikebe K. Association between oral health and sarcopenia: a literature review. J Prosthodont Res. 2021;65(2):131136. doi: 10.2186/jpr.JPOR_2019_567. PMID: 32938852. Full text links PubMed Google Scholar 15. Cruz-Jentoft AJ, Bahat G, Bauer J, et al. Sarcopenia: revised European consensus on definition and diagnosis [published correction appears in Age Ageing. 2019 Jul 1;48(4):601]. Age Ageing. 2019;48(1):16-31. doi:10.1093/ageing/afy169. Full text links Free PMC article PubMed Google Scholar WoS 16. Coelho-Junior HJ, Marzetti E, Picca A, et al. Protein intake and frailty: a matter of quantity, quality, and timing. Nutrients. 2020;12(10):2915. doi: 10.3390/nu12102915. PMID: 32977714; PMCID: PMC7598653.

Full text links Free PMC article PubMed Google Scholar WoS 17. Minakuchi S, Tsuga K, Ikebe K, et al. Oral hypofunction in the older population: position paper of the Japanese Society of Gerodontology in 2016. Gerodontology. 2018;35(4):317-324. doi: 10.1111/ger.12347. PMID: 29882364.

Full text links PubMed Google Scholar

18. Tanaka T, Takahashi $\mathrm{K}$, Hirano $\mathrm{H}$, et al. Oral frailty as a risk factor for physical frailty and mortality in community-dwelling elderly. J Gerontol A Biol Sci Med Sci. 2018;73(12):1661-1667. doi: 10.1093/gerona/glx225. PMID: 29161342.

Full text links PubMed Google Scholar WoS

19. Artaza-Artabe I, Sáez-López P, Sánchez-Hernández N, et al. The relationship between nutrition and frailty: effects of protein intake, nutritional supplementation, vitamin $D$ and exercise on muscle metabolism in the elderly. A systematic review. Maturitas. 2016;93:89-99. doi: 10.1016/j.maturitas.2016.04.009. PMID: 27125943.

Full text links PubMed Google Scholar WoS 
20. Wei K, Nyunt MSZ, Gao Q, et al. Frailty and malnutrition: related and distinct syndrome prevalence and association among community-dwelling older adults: Singapore longitudinal ageing studies. J Am Med Dir Assoc. 2017;18(12):1019-1028. doi: 10.1016/j.jamda.2017.06.017. PMID: 28804010. Full text links PubMed Google Scholar WoS 21. Verlaan S, Ligthart-Melis GC, Wijers SLJ, et al. High prevalence of physical frailty among community-dwelling malnourished older adults - a systematic review and meta-analysis. J Am Med Dir Assoc. 2017;18(5):374-382. doi: 10.1016/j.jamda.2016.12.074. PMID: 28238676.

Full text links PubMed Google Scholar WoS 22. Ligthart-Melis GC, Luiking YC, Kakourou A, et al. Frailty, sarcopenia, and malnutrition frequently (co-)occur in

hospitalized older adults: a systematic review and meta-analysis. J Am Med Dir Assoc. 2020;21(9):1216-1228. doi: 10.1016/j. jamda.2020.03.006. PMID: 32327302

Full text links PubMed Google Scholar WoS

23. Lorenzo-López L, Maseda A, de Labra C, et al. Nutritional determinants of frailty in older adults: a systematic review. BMC Geriatr. 2017;17(1):108. Published 2017 May 15. doi: 10.1186/ s12877-017-0496-2. PMID: 28506216; PMCID: PMC5433026. Full text links Free PMC article PubMed Google Scholar WoS 24. Yannakoulia M, Ntanasi E, Anastasiou CA, Scarmeas N. Frailty and nutrition: from epidemiological and clinical evidence to potential mechanisms. Metabolism. 2017;68:64-76. doi: 10.1016/j. metabol.2016.12.005. PMID: 28183454.

Full text links PubMed Google Scholar WoS

25. Filler K, Lyon D, Bennett J, et al. Association of mitochondrial dysfunction and fatigue: a review of the literature. BBA Clin. 2014;1:12-23. doi: 10.1016/j.bbacli.2014.04.001. PMCID: PMC4136529.

Full text links Free PMC article PubMed Google Scholar WoS 26. Coelho-Júnior HJ, Rodrigues B, Uchida M, Marzetti E. Low protein intake is associated with frailty in older adults: a systematic review and meta-analysis of observational studies. Nutrients. 2018;10(9):1334. doi: 10.3390/nu10091334. PMID: 30235893; PMCID: PMC6165078.

Full text links Free PMC article PubMed Google Scholar WoS 27. Shikany JM, Barrett-Connor E, Ensrud KE, et al.

Macronutrients, diet quality, and frailty in older men. J Gerontol $A$ Biol Sci Med Sci. 2014;69(6):695-701. doi: 10.1093/gerona/glt196. PMID: 24304504; PMCID: PMC4022097.

Full text links Free PMC article PubMed Google Scholar WoS 28. Chan R, Leung J, Woo J. Dietary patterns and risk of frailty in Chinese community-dwelling older people in Hong Kong: a prospective cohort study. Nutrients. 2015:7(8):7070-7084 doi: 10.3390/nu7085326. PMID: 26305253; PMCID: PMC4555165. Full text links Free PMC article PubMed Google Scholar WoS 29. Ganapathy A, Nieves JW. Nutrition and sarcopenia-what do we know? Nutrients. 2020;12(6):1755. doi: 10.3390/nu12061755. PMID: 32545408; PMCID: PMC7353446.

Full text links Free PMC article PubMed Google Scholar WoS 30. Silva R, Pizato N, da Mata F, et al. Mediterranean diet and musculoskeletal-functional outcomes in community-dwelling older people: a systematic review and meta-analysis. J Nutr Health Aging. 2018;22(6):655-663. doi: 10.1007/s12603-017-0993 1. PMID: 29806854.

Full text links PubMed Google Scholar WoS

31. Granic A, Sayer AA, Robinson SM. Dietary patterns, skeletal muscle health, and sarcopenia in older adults. Nutrients. 2019;11(4):745. doi: 10.3390/nu1 1040745. PMCID: PMC6521630. Full text links Free PMC article PubMed Google Scholar WoS 32. León-Muñoz LM, García-Esquinas E, López-García E, et al. Major dietary patterns and risk of frailty in older adults: a prospective cohort study. BMC Med. 2015;13:11. doi: 10.1186/ s12916-014-0255-6. PMID: 25601152; PMCID: PMC4298966. Full text links Free PMC article PubMed Google Scholar WoS 33. Agarwal $E$, Miller M, Yaxley A, Isenring E. Malnutrition in the elderly: a narrative review. Maturitas. 2013;76(4):296-302. doi: 10.1016/j.maturitas.2013.07.013. PMID: 23958435. Full text links PubMed Google Scholar WoS 34. Zhu Y, Hollis JH. Tooth loss and its association with dietary intake and diet quality in American adults. J Dent. 2014;42(11):1428-1435. doi: 10.1016/j.jdent.2014.08.012. PMID 25174947.

Full text links PubMed Google Scholar WoS 35. Toniazzo MP, Amorim PS, Muniz FWMG, Weidlich P. Relationship of nutritional status and oral health in elderly: systematic review with meta-analysis. Clin Nutr. 2018;37(3):824 830. doi: 10.1016/j.clnu.2017.03.014. PMID: 28392164. Full text links Google Scholar WoS

36. Gaewkhiew P, Sabbah W, Bernabé E. Does tooth loss affect dietary intake and nutritional status? A systematic review of longitudinal studies. J Dent. 2017;67:1-8. doi: 10.1016/j. jdent.2017.10.012. PMID: 29097121.

Full text links PubMed Google Scholar Wos

37. Wallace S, Samietz S, Abbas M, et al. Impact of prosthodontic rehabilitation on the masticatory performance of partially dentate older patients: can it predict nutritional state? Results from a RCT. J Dent. 2018;68:66-71. doi: 10.1016/j. jdent.2017.11.003. PMID: 29129784

Full text links PubMed Google Scholar WoS

38. Anastassiadou V. Dietary habits, nutrition and oral health. In: Kossioni A, ed. Gerodontology essentials for health care professionals. Springer Nature; 2020

Full text links Google Scholar

39. Bousiou A, Konstantopoulou K, Martimianaki G, et al. Oral factors and adherence to Mediterranean diet in an older Greek population. Aging Clin Exp Res. 2021;10.1007/s40520-021-018618. doi: 10.1007/s40520-021-01861-8. PMID: 33893988

Full text links PubMed Google Scholar WoS

40. Kossioni A, Bellou O. Eating habits in older people in Greece: the role of age, dental status and chewing difficulties. Arch Gerontol Geriatr. 2011;52(2):197-201. doi: 10.1016/j. archger.2010.03.017. PMID: 20399518.

Full text links PubMed Google Scholar WoS

41. Schimmel M, Genton L, McKenna G. Masticatory function and nutritional status: considerations for an ageing population. In Oral rehabilitation for compromised and elderly patients. Springer, Cham; 2019

Full text links Google Scholar WoS

42. Schimmel M, Katsoulis J, Genton L, Müller F. Masticatory function and nutrition in old age. Swiss Dent J. 2015;125(4):449454. https://www.sso.ch/fileadmin/upload sso/2 Zahnaerzte/2 SDJ/SDJ_2015/SDJ_Pubmed_2015/sdj-2015-04-04.pdf. PMID:26169366.

Full text links PubMed Google Scholar WoS

43. Tada A, Miura H. Systematic review of the association of mastication with food and nutrient intake in the independent elderly. Arch Gerontol Geriatr. 2014;59(3):497-505. doi: 10.1016/j. archger.2014.08.005. PMID: 25179444.

Full text links PubMed Google Scholar WoS

44. Kossioni AE. The association of poor oral health parameters with malnutrition in older adults: a review considering the potential implications for cognitive impairment. Nutrients. 2018;10(11):1709. doi: 10.3390/nu10111709

Full text links Free PMC article PubMed Google Scholar WoS 45. Laguna $L$, Chen J. The eating capability: constituents and assessments. Food Qual Prefer. 2016;48:345-358. https://www. sciencedirect.com/science/article/pii/S0950329315000683?casa token=3Fri168jMxQAAAAA:L2vGSJSOAgsfDNBBxt1wtKozOf9sLH OuGKVrHNBGKqdO-6AaQKUVH3X-ppo4BV5xC3WC8o Full text links Google Scholar WoS

46. Sura L, Madhavan A, Carnaby G, Crary MA. Dysphagia in the elderly: management and nutritional considerations. Clin Interv Aging. 2012;7:287-298. doi: 10.2147/CIA.S23404. PMID:22956864. Full text links Free PMC article PubMed Google Scholar WoS 47. Serra-Prat M, Palomera M, Gomez C, et al. Oropharyngeal dysphagia as a risk factor for malnutrition and lower respiratory tract infection in independently living older persons: a population-based prospective study. Age Ageing. 2012;41(3):376381. doi: 10.1093/ageing/afs006. PMID: 22311895.

Full text links PubMed Google Scholar WoS

48. Fávaro-Moreira NC, Krausch-Hofmann S, Matthys C, et al. Risk factors for malnutrition in older adults: a systematic review of the literature based on longitudinal data. Adv Nutr. 2016;7(3):507522. doi: 10.3945/an.115.011254. PMID: 27184278.

Full text links Free PMC article PubMed Google Scholar WoS 49. Tamura BK, Bell CL, Masaki KH, Amella EJ. Factors associated with weight loss, low BMI, and malnutrition among nursing home patients: a systematic review of the literature. J Am Med Dir Assoc. 2013;14(9):649-655. doi: 10.1016/j.jamda.2013.02.022. PMID: 23639716

Full text links PubMed Google Scholar WoS

50. Quandt SA, Savoca MR, Leng X, et al. Dry mouth and dietary quality in older adults in North Carolina. J Am Geriatr Soc. 2011;59(3):439-445. doi: 10.1111/j.1532-5415.2010.03309.x. PMID: 21391935; PMCID: PMC3096847.

Full text links PMC free article PubMed Google Scholar WoS 51. Iwasaki M, Motokawa K, Watanabe Y, et al. Association between oral frailty and nutritional status among communitydwelling older adults: the Takashimadaira study. J Nutr Health Aging. 2020;24(9):1003-1010. doi: 10.1007/s12603-020-1433-1. PMID: 33155629.

Full text links $\underline{\text { PubMed Google Scholar WoS }}$ 
52. Iwasaki M, Motokawa K, Watanabe Y, et al. A two-year longitudinal study of the association between oral frailty and deteriorating nutritional status among community-dwelling older adults. Int J Environ Res Public Health. 2020;18(1):213. Published 2020 Dec 30. doi: 10.3390/ijerph18010213. PMID: 33396639; PMCID: PMC7796237.

Full text links Free PMC article PubMed Google Scholar WoS 53. Van Lancker A, Verhaeghe S, Van Hecke A, et al. The association between malnutrition and oral health status in elderly in long-term care facilities: a systematic review. Int J Nurs Stud. 2012;49(12):1568-1581. doi: 10.1016/j.ijnurstu.2012.04.001. PMID: 22542267

Full text links PubMed Google Scholar WoS

54. O'Keeffe M, Kelly M, O'Herlihy E, et al. Potentially modifiable determinants of malnutrition in older adults: a systematic review. Clin Nutr. 2019;38(6):2477-2498. doi: 10.1016/j.clnu.2018.12.007. PMID: 30685297.

Full text links PubMed Google Scholar WoS

55. Franceschi C, Campisi J. Chronic inflammation (inflammaging) and its potential contribution to age-associated diseases. $J$ Gerontol A Biol Sci Med Sci. 2014;69 Suppl 1:S4-S9. doi: 10.1093/ gerona/glu057. PMID: 24833586.

Full text links PubMed Google Scholar WoS

56. Marcos-Pérez D, Sánchez-Flores M, Proietti S, et al. Association of inflammatory mediators with frailty status in older adults: results from a systematic review and meta-analysis. Geroscience. 2020;42(6):1451-1473. doi:10.1007/s11357-020-00247-4. PMID: 32803650; PMCID: PMC7732924.

Full text links Free PMC article PubMed Google Scholar WoS 57. Michaud M, Balardy L, Moulis G, et al. Proinflammatory cytokines, aging, and age-related diseases. J Am Med Dir Assoc. 2013;14(12):877-882. doi: 10.1016/j.jamda.2013.05.009. PMID: 23792036

Full text links PubMed Google Scholar WoS

58. Soysal $P$, Stubbs B, Lucato $P$, et al. Inflammation and frailty in the elderly: a systematic review and meta-analysis. Ageing Res Rev. 2016;31:1-8. doi: 10.1016/j.arr.2016.08.006. PMID: 27592340. Full text links PubMed Google Scholar WoS

59. Verghese J, Holtzer R, Oh-Park M, et al. Inflammatory markers and gait speed decline in older adults. J Gerontol A Biol Sci Med Sci. 2011;66(10):1083-1089. doi: 10.1093/gerona/glr099. PMID: 21719612: PMCID: PMC3202897.

Full text links PubMed Free PMC article Google Scholar WoS 60. Tuttle CSL, Thang LAN, Maier AB. Markers of inflammation and their association with muscle strength and mass: a systematic review and meta-analysis. Ageing Res Rev. 2020;64:101185. doi: 10.1016/j.arr.2020.101185. PMID: 32992047.

Full text links PubMed Google Scholar WoS

61. Bano G, Trevisan C, Carraro S, et al. Inflammation and sarcopenia: a systematic review and meta-analysis. Maturitas. 2017;96:10 15. doi: 10.1016/j.maturitas.2016.11.006. PMID: 28041587. Full text links PubMed Google Scholar WoS 62. Cecoro G, Annunziata M, luorio MT, et al. Periodontitis, lowgrade inflammation and systemic health: a scoping review. Medicina (Kaunas). 2020;56(6):272. doi: 10.3390/medicina56060272. PMID: 32486269; PMCID: PMC7353850. Full text links Free PMC article PubMed Google Scholar WoS 63. Buigues C, Padilla-Sánchez C, Garrido JF, et al. The relationship between depression and frailty syndrome: a systematic review. Aging Ment Health. 2015;19(9):762-772. doi: 10.1080/13607863.2014.967174. PMID: 25319638.

Full text links PubMed Google Scholar WoS

64. Soysal P, Veronese $N$, Thompson T, et al. Relationship between depression and frailty in older adults: a systematic review and meta-analysis. Ageing Res Rev. 2017;36:78-87. doi: 10.1016/j. arr.2017.03.005. PMID: 28366616.

Full text links PubMed Google Scholar WoS

65. Vaughan L, Corbin AL, Goveas JS. Depression and frailty in later life: a systematic review. Clin Interv Aging. 2015:10:1947-1958. doi: 10.2147/CIA.S69632. PMID: 26719681; PMCID: PMC4687619. Free PMC article PubMed Google Scholar WoS

66. Chu W, Chang SF, Ho HY, Lin HC. The relationship between depression and frailty in community-dwelling older people: a systematic review and meta-analysis of 84,351 older adults. J Nurs Scholarsh. 2019;51(5):547-559. doi: 10.1111/jnu.12501. PMID: 31328878.

Full text links PubMed Google Scholar WoS

67. Zasadzka E, Pieczyńska A, Trzmiel T, et al. Correlation between handgrip strength and depression in older adults-a systematic review and a meta-analysis. Int J Environ Res Public Health. 2021;18(9):4823. doi: 10.3390/ijerph18094823. PMID: 33946502; PMCID: PMC8124581.

Full text links Free PMC article PubMed Google Scholar WoS
68. Davies K, Maharani A, Chandola T, et al. The longitudinal relationship between loneliness, social isolation, and frailty in older adults in England: a prospective analysis. Lancet Healthy Longev. 2021;2(2):70-77. https://www.sciencedirect.com/science/ article/pii/S2666756820300386.

Full text links Google Scholar

69. Tamada Y, Takeuchi K, Yamaguchi C, et al. Does laughter predict onset of functional disability and mortality among older Japanese adults? The JAGES prospective cohort study.

J Epidemiol. 2021;31(5):301-307. doi: 10.2188/jea.JE20200051. PMID: 32418940; PMCID: PMC8021882.

Full text link Free PMC article PubMed Google Scholar WoS

70. Gale CR, Westbury L, Cooper C. Social isolation and loneliness as risk factors for the progression of frailty: the English longitudinal study of ageing. Age Ageing. 2018;47(3):392-397. doi: 10.1093/ ageing/afx188. PMID: 29309502; PMCID: PMC5920346.

Full text article Free PMC article PubMed Google Scholar WoS 71. Da Mata FAF, Miranda Forte Gomes M, Lício Ferreira Santos J, et al. Depression and frailty in older adults: a population-based cohort study. PLoS One. 2021;16(3):e0247766. doi: 10.1371/journal.pone.0247766. MID: 33662006; PMCID: PMC7932072. Full text links Free PMC article PubMed Google Scholar WoS 72. Mayerl H, Stolz E, FreidI W. Frailty and depression: reciprocal influences or common causes? Soc Sci Med. 2020;263:113273. doi: 10.1016/j.socscimed.2020.113273. PMID: 32810695.

Full text links PubMed Google Scholar WoS

73. Kossioni AE, Dontas AS. The stomatognathic system in the elderly. Useful information for the medical practitioner. Clin Interv Aging. 2007;2(4):591-597. doi: 10.2147/cia.s1596. MID: 18225459; PMCID: PMC2686331.

Full text links Free PMC article PubMed Google Scholar 74. Kisely S, Sawyer E, Siskind D, Lalloo R. The oral health of people with anxiety and depressive disorders - a systematic review and meta-analysis. J Affect Disord. 2016;200:119-132. doi: 10.1016/j.jad.2016.04.040. PMID: 27130961.

Full text links PubMed Google Scholar WoS

75. Kandelman D, Petersen PE, Ueda H. Oral health, general health, and quality of life in older people. Spec Care Dentist. 2008;28(6):224-236. doi: 10.1111/j.1754-4505.2008.00045.x. PMID: 19068063

Full text links PubMed Google Scholar WoS

76. Rouxel $P$, Heilmann A, Demakakos $P$, et al. Oral health-related quality of life and loneliness among older adults. Eur J Ageing. 2016:14(2):101-109 doi: 10.1007/s10433-016-0392-1. PMID: 28579932; PMCID: PMC5435788.

Full text links Free PMC article PubMed Google Scholar WoS 77. Niesten D, van Mourik K, van der Sanden W. The impact of having natural teeth on the QoL of frail dentulous older people. A qualitative study. BMC Public Health. 2012;12:839. doi: 10.1186/1471-2458-12-839. PMID: 23031489; PMCID: PMC3524040.

Full text links Free PMC article PubMed Google Scholar WoS 78. Koyama S, Saito M, Cable N, et al. Examining the associations between oral health and social isolation: a cross-national comparative study between Japan and England. Soc Sci Med. 2021;277:113895. doi: 10.1016/j.socscimed.2021.113895. PMID: 33882441.

Full text links PubMed Google Scholar

79. Kunrath I, Silva AER. Oral health and depressive symptoms among older adults: Iongitudinal study. Aging Ment Health. 2020;1-7. doi: 10.1080/13607863.2020.1855104. PMID: 33291966. Full text links PubMed Google Scholar WoS

80. Yamamoto T, Aida J, Kondo K, et al. Oral health and incident depressive symptoms: JAGES project longitudinal study in older Japanese. J Am Geriatr Soc. 2017;65(5):1079-1084. doi: 10.1111/ jgs.14777. PMID: 28165614

Full text links PubMed Google Scholar WoS

81. Gkavela G, Kossioni A, Lyrakos G, et al. Oral health related quality of life in older people: preliminary validation of the Greek version of the Geriatric Oral Health Assessment Index (GOHAI). Eur Geriatr Med. 2015;6(3):245-250. https://www.sciencedirect. $\mathrm{com} /$ science/article/pii/S1878764915000030?casa_token=jriqF tyLf_kAAAAA:6EwCjjZ8Lz_IDjmy76clg4xk3QaGmn1L0ZIG7V76EXkYpjgbk4NF7o52B4VWMIHVNFN0yiM.

Full text links Google Scholar WoS

82. Cademartori MG, Gastal MT, Nascimento GG, et al. Is depression associated with oral health outcomes in adults and elders? A systematic review and meta-analysis. Clin Oral Investig. 2018;22(8):2685-2702. doi: 10.1007/s00784-018-2611-y. PMID: 30191327

Full text links $\underline{\text { PubMed Google Scholar WoS }}$ 
83. Hirosaki M, Ohira T, Shirai K, et al. Association between frequency of laughter and oral health among community-dwelling older adults: a population-based cross-sectional study in Japan. Qual Life Res. 2021;30(6):1561-1569. doi: 10.1007/s11136-020-

02752-7. PMID: 33432445.

Full text links PubMed Google Scholar WoS

84. Hsu CC, Hsu YC, Chen $\mathrm{HJ}$, et al. Association of periodontitis and subsequent depression: a nationwide population-based study. Medicine (Baltimore). 2015;94(51):e2347. doi: 10.1097/ MD.0000000000002347. PMID: 26705230; PMCID: PMC4697996. Free PMC article PubMed Google Scholar WoS

85. Kossioni A, Bellou O. The effect of aging and dental status on the frequency of eating out. Arch Gerontol Geriatr. 2012;54(2):e130-e133. doi: 10.1016/j.archger.2011.10.016. PMID: 22088937.

Full text links PubMed Google Scholar WoS

86. Cuevas-Trisan R. Balance problems and fall risks in the elderly. Phys Med Rehabil Clin N Am. 2017;28(4):727-737. doi: 10.1016/j. pmr.2017.06.006. PMID: 29031339.

Full text links PubMed Google Scholar WoS

87. Chkeir A, Safieddine D, Bera D, et al. Balance quality assess-

ment as an early indicator of physical frailty in older people. Annu Int Conf IEEE Eng Med Biol Soc. 2016;2016:5368-5371. doi: 10.1109/ EMBC.2016.7591940. PMID: 28325024.

Full text links PubMed Google Scholar WoS

88. Moraes DC, Lenardt MH, Seima MD, et al. Postural instability and the condition of physical frailty in the elderly. Rev Lat Am Enfermagem. 2019;27:e3146. doi: 10.1590/1518-8345.2655-3146. PMID: 31038639; PMCID: PMC6528635.

Full text links Free PMC article PubMed Google Scholar WoS 89. Fhon JR, Rodrigues RA, Neira WF, et al. Fall and its association with the frailty syndrome in the elderly: systematic review with meta-analysis. Rev ESC Enferm USP. 2016;50(6):1005-1013. doi: 10.1590/S0080-623420160000700018. PMID: 28198967. Full text links PubMed Google Scholar WoS

90. Kojima G. Frailty as a predictor of future falls among commu nity-dwelling older people: a systematic review and meta-analysis. J Am Med Dir Assoc. 2015;16(12):1027-1033. doi: 10.1016/j. jamda.2015.06.018. PMID: 26255098.

Full text links PubMed Google Scholar WoS

91. Cheng $\mathrm{MH}$, Chang SF. Frailty as a risk factor for falls among community dwelling people: evidence from a meta-analysis.

J Nurs Scholarsh. 2017;49(5):529-536. doi: 10.1111/jnu.12322.

PMID: 28755453.

Full text links PubMed Google Scholar WoS

92. Cuccia A, Caradonna C. The relationship between the stomatognathic system and body posture. Clinics (Sao Paulo). 2009;64(1):61-66. doi: 10.1590/s1807-59322009000100011. PMID: 19142553; PMCID: PMC2671973.

Full text links Free PMC article PubMed Google Scholar WoS
93. Yoshida M, Kikutani T, Okada G, et al. The effect of tooth loss on body balance control among community-dwelling elderly persons. Int J Prosthodont. 2009;22(2):136-

139. https://web.b.ebscohost.com/abstract?direct=true\&profile $=$ ehost\&scope $=$ site\&authtype $=$ crawler\&jrnI=08932174\&AN=43288454\&h=Ap2\%2fiS9jqUyBz2CIFIrliR7Q4Egb5WqHg348oyjgEicKoBGtgm0TB0prjNnQfDGVr-

MgEe8WfY8fs9QjBvx\%2bGlg\%3d\%3d\&crl=c\&resultNs=AdminWebAuth\&resultLocal=ErrCrINo-

tAuth\&crlhashurl=login.aspx\%3fdirect $\% 3$ dtrue $\% 26$ pro-

file $\% 3 d$ ehost $\% 26$ scope $\% 3 d$ site $\% 26$ authtype $\% 3 d$ crawler $\% 26 j r n-$ \%3d08932174\%26AN\%3d43288454. PMID: 19418857.

Full text links PubMed Google Scholar WoS

94. Okubo M, Fujinami Y, Minakuchi S. Effect of complete dentures on body balance during standing and walking in elderly people. J Prosthodont Res. 2010;54(1):42-47. doi: 10.1016/j. jpor.2009.09.002. PMID: 19819207.

Full text links PubMed Google Scholar

95. Okuyama N, Yamaga T, Yoshihara A, et al. Influence of dental occlusion on physical fitness decline in a healthy Japanese elderly population. Arch Gerontol Geriatr. 2011;52(2):172-176. doi: 10.1016/j.archger.2010.03.011. PMID: 20378189.

Full text links PubMed Google Scholar PubMed

96. linuma T, Arai Y, Fukumoto M, et al. Maximum occlusal force and physical performance in the oldest old: the Tokyo oldest old survey on total health. J Am Geriatr Soc. 2012;60(1):68-76. doi:10.1111/j.1532-5415.2011.03780.x

Full text links PubMed Google Scholar

97. Song-Yu X, Rodis OM, Ogata S, et al. Postural stability and occlusal status among Japanese elderly. Gerodontology.

2012;29(2):e988-e997. doi:10.1111/j.1741-2358.2011.00596.x

Full text links PubMed Google Scholar

98. Yamamoto T, Kondo K, Misawa J, et al. Dental status and incident falls among older Japanese: a prospective cohort study. BMJ Open. 2012;2(4):e001262. doi: 10.1136/bmjopen-2012-001262. PMID: 22855628; PMCID: PMC4400665.

Full text links Free PMC article PubMed Google Scholar WoS 99. Eshkoor SA, Hamid TA, Nudin SS, Mun CY. Association between dentures and the rate of falls in dementia. Med Devices (Auckl). 2014;7:225-230. Published 2014 Jun 20. doi:10.2147/ MDER.S63220

Full text links Free PMC article PubMed Google Scholar 100. Bae Y, Park Y. Head posture and postural balance in community-dwelling older adults who use dentures. Medicina (Kaunas). 2020;56(10):529. Published 2020 Oct 12. doi:10.3390/medicina56100529

Full text links Free PMC article PubMed Google Scholar

\title{
Kalliopi KONSTANTOPOULOU
}

\author{
DDS, MSc \\ Department of Prosthodontics \\ School of Dentistry \\ National and Kapodistrian University of Athens \\ Athens, Greece
}

Kalliopi Konstantopoulou graduated from the Dental School of National and Kapodistrian University of Athens, Athens, Greece in 2016. In 2019, she obtained her master's degree in Health Promotion and Education from the Medical School of National and Kapodistrian University of Athens and is currently a postgraduate student at the Department of Prosthodontics, Dental School, National and Kapodistrian University of Athens, Athens, Greece. She is also a Board member at the European College of Gerodontology. 


\title{
Questions
}

\author{
1. The most widely used instrument to assess frailty is: \\ $\square$ a. Physical Frailty Phenotype; \\ ab. Frailty Index; \\ Dc. Clinical Frailty Scale; \\ Dd. Short Physical Performance Battery.
}

\section{Oral health is linked to frailty through:}

a. Nutrition;

Db. Inflammation;

ac. Psychological and neural pathways;

$\square$ d. All of the above are correct.

\section{Which of the following sentences is correct?}

$\square$ a. Oral health is the dominant determinant of malnutrition;

ab. Nutritional status and food selection have a multifactorial nature;

口c. Malnutrition can be reversed solely by prosthodontic rehabilitation;

$\square$ d. Westernized dietary patterns may prevent frailty.

\section{Which of the following sentences is correct?}

$\square$ a. No prospective associations exist between depression and frailty;

$\square$ b. No reciprocal associations exist between depression and frailty;

D. Depression and frailty seem to be affected by common causes;

Dd. None of the above.

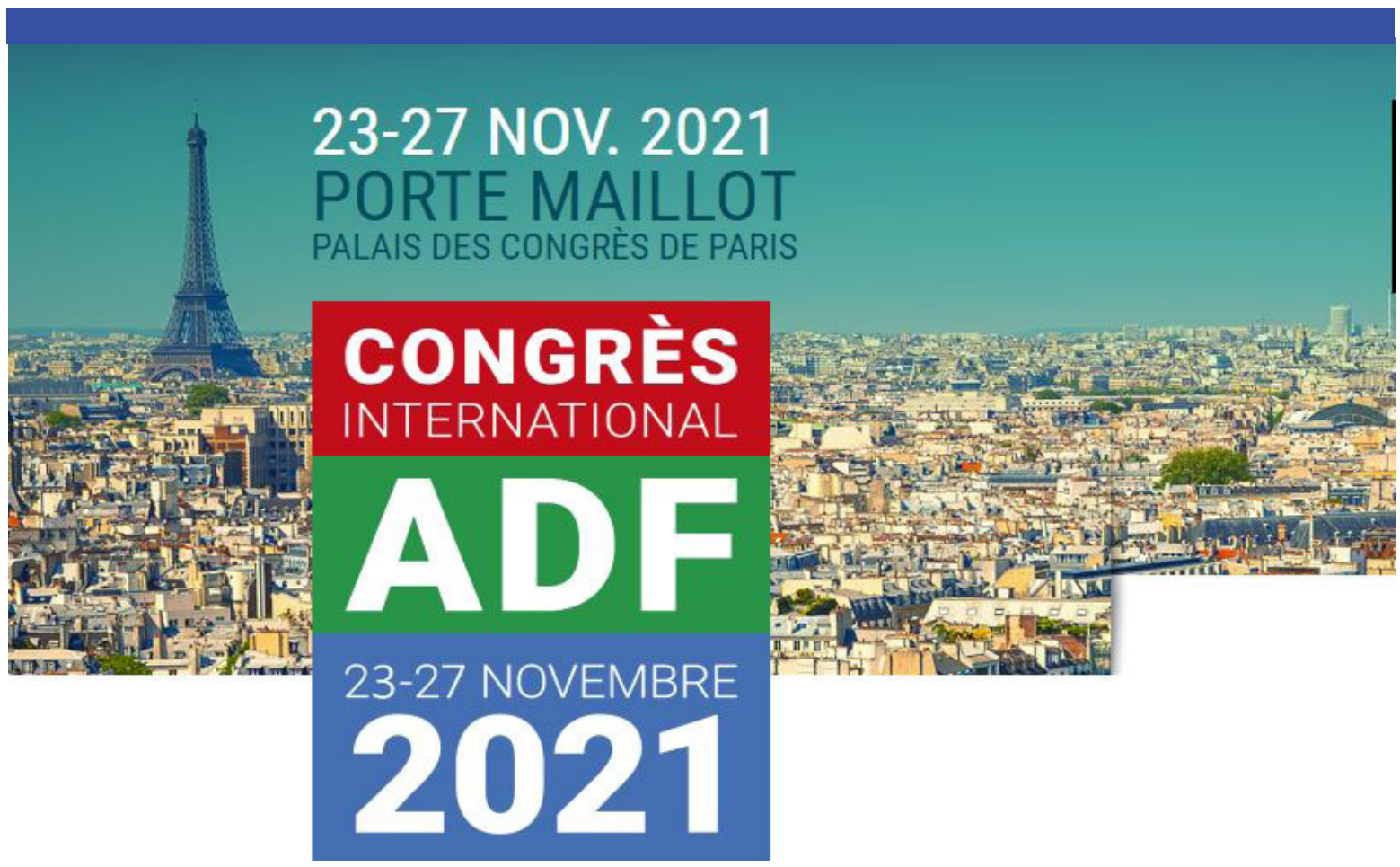

